\title{
ON THE ORDER AND THE LOWER ORDER OF DIFFERENTIAL POLYNOMIALS
}

\author{
M. N. KULKARNI AND KIT-WING YU
}

\begin{abstract}
Suppose that $f$ is a meromorphc function with order $\sigma(f)$ and lower order $\mu(f)$. Suppose that $P[f]$ is a differential polynomial of $f$. In this paper, it is shown that the order and the lower order of $P[f]$ are equal to the order and the lower order of $f$ under certain conditions on the degree of the differential polynomial $P[f]$, i.e., $\sigma(P)=\sigma(f)$ and $\mu(P)=\mu(f)$. This result improves previous results.
\end{abstract}

\section{Introduction}

Let $f$ be a transcendental meromorphic function in the complex plane $\mathbb{C}$. It is assumed that the reader is familiar with the usual notations, such as $m(r, f), N(r, f), \bar{N}(r, f), T(r, f)$ and etc., of Nevanlinna theory, see e.g., [4, 9]. We denote by $S(r, f)$ any quantity satisfying $S(r, f)=o(T(r, f))$ as $r \rightarrow+\infty$, possibly outside a set of finite linear measure $E$. Throughout this paper we denote by $a_{j}$ any small meromorphic function satisfying $T\left(r, a_{j}\right)=S(r, f)$ with $j=1,2, \ldots, n$. As usual, the order $\sigma(f)$ and the lower order $\mu(f)$ of $f$ are defined by

$$
\sigma(f):=\limsup _{r \rightarrow+\infty} \frac{\log T(r, f)}{\log r} \text { and } \mu(f):=\liminf _{r \rightarrow+\infty} \frac{\log T(r, f)}{\log r}
$$

respectively.

There has been quite a number of researches on the value distribution of differential polynomials since the 1940s. For example, H. Milloux [5] (see also [4, Theorem 3.3]) showed in 1940 that if $f$ is a transcendental meromorphic function having only a finite number of zeros and poles, then the function $\psi$ defined by

$$
\psi:=\sum_{j=0}^{n} a_{j} f^{(j)}
$$

assumes every finite complex value except possibly zero infinitely many times or else $\psi$ is

Corresponding author: Milind-Narayanrao Kulkarni.

2010 Mathematics Subject Classification. 30D30, 30D35.

Key words and phrases. Differential polynomials, homogeneous, lower order, meromorphic functions, Nevanlinna theory, non-homogeneous, order. 
identically constant, where $a_{j}$ are small functions of $f$ with $j=0,1, \ldots, n$. Another example is given by W. K. Hayman. In fact, he [3] (see also [4, Theorem 3.5]) showed in 1959 that if $f$ is a transcendental meromorphic function, then either $f$ assumes every finite value infinitely many times or every derivative of $f$ assumes every finite value except possibly zero infinitely many times.

We note that their results are true no matter what the growth of the meromorphic function $f$ is. However, if we restrict its growth, then people can obtain other kind of results of the function $f$ and its differential polynomial $P[f]$. Specifically, mathematicians have paid attention to the study of the relations between the order and the lower order of the transcendental meromoprhic function $f$ and its differential polynomials $P[f]$. In particular, L. R. Sons [8, Theorem 3] showed in 1969 that if $f$ is a transcendental meromorphic function of finite order $\sigma$ and lower order $\mu$, then $\sigma(f)=\sigma(P)$ and $\mu(f)=\mu(P)$, where $P[f]$ is a monomial given by

$$
P[f]=(f)^{n_{0}}\left(f^{\prime}\right)^{n_{1}} \cdots\left(f^{(k)}\right)^{n_{k}},
$$

$n_{0} \geq 1, n_{k} \geq 1, n_{i} \geq 0$ for $1 \leq i \leq k-1$. Later in 1985, A. P. Singh [6] generalized L. R. Sons' result to functions of infinite order and also to a class of homogeneous differential polynomial $P[f]$. In fact, he showed that

Theorem A. Let $f$ be a transcendental meromorphic function and let $P[f]$ be a non-zero homogeneous differential polynomial of degree $n$ in the form

$$
\begin{aligned}
P[f]= & a_{0}(f)^{v_{0}}\left(f^{\prime}\right)^{v_{1}} \cdots\left(f^{(i)}\right)^{v_{i}}+b_{0}(f)^{\mu_{0}}\left(f^{\prime}\right)^{\mu_{1}} \cdots\left(f^{(j)}\right)^{\mu_{j}} \\
& +\cdots+c_{0}(f)^{\delta_{0}}\left(f^{\prime}\right)^{\delta_{1}} \cdots\left(f^{(k)}\right)^{\delta_{k}}
\end{aligned}
$$

satisfying that each of the exponents of $f$, i.e., $v_{0}, \mu_{0}, \ldots, \delta_{0}$, is an integer greater than or equal to 1 and $a_{0}, b_{0}, \ldots, c_{0}$ are small functions of $f$. Then we have $\sigma(P)=\sigma(f)$.

In this direction, A. P. Singh and R. S. Dhar [7, Theorem 1] enriched the Theorem A to

Theorem B. Let $f$ be a transcendental meromorphic function and $P[f]$ be a non-constant differential polynomial of degree $\bar{d}(P)$ in the form

$$
P[f]:=a+Q[f]=a+\sum_{j=1}^{n} a_{j}(f)^{l_{0 j}}\left(f^{\prime}\right)^{l_{1 j}} \cdots\left(f^{(k)}\right)^{l_{k j}}
$$

where $T(r, a)=S(r, f)$, each of the exponents of $f$ is an integer greater than or equal to 1 and $\sum_{j=1}^{n} d\left(M_{j}\right)>(n-1) \bar{d}(Q)$. Then we must have $\sigma(P)=\sigma(f)$.

This paper is organized as follows: In $\$ 2$, we give a very brief review on the differential polynomials of a meromorphic function $f$. After that, the main results of this paper are stated. 
In $\S 3$, the necessary lemmas are stated and proofs of our main results are given in $\S 4$. In $\$ 5$, two remarks about our main results and previous results are given.

\section{Definitions and the main results}

This paper concerns the value distribution of the differential polynomials of a meromorphic function $f$, so we shall give a brief review to the definitions of the study here. For a positive integer $j$, by a monomial in $f$ we mean an expression of the type

$$
M_{j}[f]=a_{j}(f)^{n_{0 j}}\left(f^{\prime}\right)^{n_{1 j}} \cdots\left(f^{(k)}\right)^{n_{k j}},
$$

where $n_{0 j}, n_{1 j}, \ldots, n_{k j}$ are non-negative integers. We define $d\left(M_{j}\right)=\sum_{i=0}^{k} n_{i j}$ as the degree of $M_{j}[f]$ and $\Gamma_{M_{j}}=\sum_{i=0}^{k}(i+1) n_{i j}$ as the weight of $M_{j}[f]$. Next a differential polynomial in $f$ is a finite sum of such monomials (2.1), i.e.,

$$
P[f]=\sum_{j=1}^{n} a_{j} M_{j}[f] .
$$

We define

$$
\bar{d}(P)=\max _{1 \leq j \leq n}\left\{d\left(M_{j}\right)\right\}, \quad \underline{d}(P)=\min _{1 \leq j \leq n}\left\{d\left(M_{j}\right)\right\} \quad \text { and } \quad \Gamma_{P}=\max _{1 \leq j \leq n}\left\{\Gamma_{M_{j}}\right\}
$$

as the degree, the lower degree and the weight of $P[f]$, respectively. If, in particular, $\bar{d}(P)=$ $\underline{d}(P)$, then $P[f]$ is called homogeneous and non-homogeneous otherwise.

We have two main results which are stated as follows:

Theorem 2.1. Suppose that $f$ is a transcendental meromorphic function and $P[f]$ is a nonconstant differential polynomial (2.2) with $T\left(r, a_{j}\right)=S(r, f)$, where $j=1,2, \ldots, n$, satisfying that each $n_{j}$ of the exponents of $f$ is a positive integer. If the constant

$$
d:=\sum_{j=1}^{n} d\left(M_{j}\right)-(n-1)[\bar{d}(P)-\underline{d}(P)]>0,
$$

then we must have $\sigma(P)=\sigma(f)$ and $\mu(P)=\mu(f)$.

Theorem 2.2. Suppose that $f$ is a meromorphic function satisfying the condition

$$
\bar{N}(r, f)+\bar{N}\left(r, \frac{1}{f}\right)=S(r, f) .
$$

If $P[f]$ is a non-constant differential polynomial, each term in $P[f]$ contains $f$ and $d>0$, then we have

$$
\sigma(P)=\sigma(f), \mu(P)=\mu(f) \quad \text { and } \quad \bar{N}(r, P)+\bar{N}\left(r, \frac{1}{P}\right)=S(r, P) .
$$




\section{Lemmas}

For the proofs of our main results, we need the following two lemmas due to W. Doeringer.

Lemma 3.1. [1, Lemma 1] Let $f$ be a non-constant meromorphic function. If $P[f]$ is a differential polynomial in $f$ with arbitrary meromorphic coefficients $a_{j}, 1 \leq j \leq n$, then we have

$$
m(r, P) \leq \bar{d}(P) m(r, f)+\sum_{j=1}^{n} m\left(r, a_{j}\right)+S(r, f)
$$

and

$$
N(r, P) \leq \Gamma_{P} N(r, f)+\sum_{j=1}^{n} N\left(r, a_{j}\right)+O(1)
$$

Lemma 3.2. [1, Lemma 3] Let $T_{1}(r)$ and $T_{2}(r)$ be real valued, non-negative and non-decreasing functions defined for $r>r_{0}>0$ and satisfying $T_{1}(r)=O\left(T_{2}(r)\right)$ as $r \rightarrow+\infty$. Then we have

$$
\limsup _{r \rightarrow+\infty} \frac{\log ^{+} T_{1}(r)}{\log r} \leq \limsup _{r \rightarrow+\infty} \frac{\log ^{+} T_{2}(r)}{\log r}
$$

and

$$
\liminf _{r \rightarrow+\infty} \frac{\log ^{+} T_{1}(r)}{\log r} \leq \liminf _{r \rightarrow+\infty} \frac{\log ^{+} T_{2}(r)}{\log r} .
$$

In particular, this implies that for meromorphic functions $f_{1}$ and $f_{2}$ with $T\left(r, f_{1}\right)=O\left(T\left(r, f_{2}\right)\right)$, $r \rightarrow+\infty$, possibly outside a set of finite linear measure, the inequalities $\mu\left(f_{1}\right) \leq \mu\left(f_{2}\right)$ and $\sigma\left(f_{1}\right) \leq \sigma\left(f_{2}\right)$ hold .

\section{Proofs of the main results}

\subsection{Proof of Theorem 2.1.}

By the Lemma 3.1 and the fact that $\bar{d}(P) \leq \Gamma_{P}$, we have

$$
T(r, P) \leq \Gamma_{P} T(r, f)+S(r, f) .
$$

Since $P[f]$ is non-constant, we have $P[f] \not \equiv 0$ and then

$$
\frac{1}{f^{\bar{d}(P)-\underline{d}(P)}}=\frac{1}{P[f]} \cdot \frac{P[f]}{f^{\bar{d}(P)-\underline{d}(P)}}
$$

which gives

$$
T\left(r, \frac{1}{f^{\bar{d}(P)-\underline{d}(P)}}\right) \leq T\left(r, \frac{1}{P}\right)+T\left(r, \sum_{j=1}^{n} \frac{a_{j} M_{j}[f]}{f^{d\left(M_{j}\right)}} \cdot \frac{f^{d\left(M_{j}\right)}}{f^{\bar{d}(P)-\underline{d}(P)}}\right) .
$$


Thus the inequality (4.2) together with the First Fundamental Theorem imply that

$$
\begin{aligned}
& {[\bar{d}(P)-\underline{d}(P)] T(r, f) \leq T\left(r, \frac{1}{f^{\bar{d}(P)-\underline{d}(P)}}\right)+O(1)} \\
& \leq T(r, P)+\sum_{j=1}^{n} T\left(r, \frac{a_{j} M_{j}[f]}{f^{d\left(M_{j}\right)}}\right) \\
& +\sum_{j=1}^{n} T\left(r, \frac{1}{f^{\bar{d}}(P)-\underline{d}(P)-d\left(M_{j}\right)}\right)+O(1)
\end{aligned}
$$

which deduces that

$$
d T(r, f) \leq T(r, P)+\sum_{j=1}^{n} T\left(r, \frac{M_{j}[f]}{f^{d\left(M_{j}\right)}}\right)+S(r, f) .
$$

Now for all positive integers $p$, we have

$$
N\left(r, \frac{f^{(p)}}{f}\right) \leq p\left[\bar{N}(r, f)+\bar{N}\left(r, \frac{1}{f}\right)\right] \leq p\left[\bar{N}(r, P)+\bar{N}\left(r, \frac{1}{P}\right)\right]
$$

because each of $n_{0 j}$ is an integer $\geq 1$, where $j=1,2, \ldots, n$. Furthermore, we have

$$
\begin{aligned}
m\left(r, \frac{M_{j}[f]}{f^{d\left(M_{j}\right)}}\right) & =m\left(r, \frac{f^{n_{0 j}}\left(f^{\prime}\right)^{n_{1 j}} \cdots\left(f^{(k)}\right)^{n_{k j}}}{f^{\sum_{j=0}^{k} n_{i j}}}\right) \\
& =m\left(r, \frac{f^{n_{0 j}}\left(f^{\prime}\right)^{n_{1 j}} \cdots\left(f^{(k)}\right)^{n_{k j}}}{f^{n_{0 j}} \cdot f^{n_{1 j}} \cdots f^{n_{k j}}}\right) \\
& =S(r, f)
\end{aligned}
$$

and

$$
\begin{aligned}
N\left(r, \frac{M_{j}[f]}{f^{d\left(M_{j}\right)}}\right) & =n_{1 j} N\left(r, \frac{f^{\prime}}{f}\right)+n_{2 j} N\left(r, \frac{f^{\prime \prime}}{f}\right)+\cdots+n_{k j} N\left(r, \frac{f^{(k)}}{f}\right) \\
& \leq\left(n_{1 j}+2 n_{2 j}+\cdots+k n_{k j}\right)\left[\bar{N}(r, P)+\bar{N}\left(r, \frac{1}{P}\right)\right] .
\end{aligned}
$$

Thus we have

$$
T\left(r, \frac{M_{j}[f]}{f^{d\left(M_{j}\right)}}\right) \leq 2\left[\Gamma_{M_{j}}-d\left(M_{j}\right)\right] T(r, P)+S(r, P)+S(r, f) .
$$

Hence inequalities (4.4) and (4.8) imply that

$$
d T(r, f) \leq T(r, P)+\sum_{j=1}^{n} 2\left[\Gamma_{M_{j}}-d\left(M_{j}\right)\right] T(r, P)+S(r, P)+S(r, f) .
$$

By (4.1), we must have $S(r, P)=S(r, f)$ so that

$$
d T(r, f) \leq d_{1} T(r, P)+S(r, f)
$$


where $d_{1}=2 \sum_{j=1}^{n}\left[\Gamma_{M_{j}}-d\left(M_{j}\right)\right]+1>0$. Since $d>0$ by hypothesis, it follows that

$$
T(r, f) \leq d_{2} T(r, P)+S(r, f),
$$

where $d_{2}=\frac{d_{1}}{d}$. Hence inequalities (4.1), (4.10) and the Lemma 3.2 show that

$$
\sigma(P)=\sigma(f) \text { and } \mu(P)=\mu(f)
$$

as required.

\subsection{Proof of Theorem 2.2}

By the condition (2.4), we have from the estimate (4.5) that

$$
N\left(r, \frac{f^{(p)}}{f}\right)=S(r, f)
$$

for integers $p$. Thus the inequality (4.7) gives

$$
N\left(r, \frac{M_{j}[f]}{f^{d\left(M_{j}\right)}}\right)=S(r, f)
$$

for each $j=1,2, \ldots, n$ and combining (4.6), we obtain that

$$
T\left(r, \frac{M_{j}[f]}{f^{d\left(M_{j}\right)}}\right)=S(r, f)
$$

for each $j=1,2, \ldots, n$. Therefore it follows from (4.4) that

$$
d T(r, f) \leq T(r, P)+S(r, f)
$$

where $d$ is defined by (2.3). By the inequalities (4.1), (4.12) and the Lemma 3.2, we have

$$
\sigma(f)=\sigma(P) \text { and } \quad \mu(f)=\mu(P)
$$

as required.

Furthermore, since $\bar{N}(P) \leq \bar{N}(r, f)+S(r, f)$, the condition (2.4) implies that

$$
\bar{N}(P)=S(r, f)=S(r, P)
$$

and for any $j$,

$$
\begin{aligned}
\bar{N}\left(r, \frac{1}{P}\right) & \leq \bar{N}\left(r, \frac{f^{d\left(M_{j}\right)}}{P}\right)+\bar{N}\left(r, \frac{1}{f^{d\left(M_{j}\right)}}\right) \\
& \leq T\left(r, \frac{f^{d\left(M_{j}\right)}}{P}\right)+\bar{N}\left(r, \frac{1}{f^{d\left(M_{j}\right)}}\right)
\end{aligned}
$$




$$
\begin{aligned}
& \leq T\left(r, \frac{P}{f^{d\left(M_{j}\right)}}\right)+\bar{N}\left(r, \frac{1}{f}\right)+O(1) \\
& \leq \sum_{j=1}^{n} T\left(r, \frac{M_{j}[f]}{f^{d\left(M_{j}\right)}}\right)+\bar{N}\left(r, \frac{1}{f}\right)+O(1),
\end{aligned}
$$

so that

$$
\bar{N}\left(r, \frac{1}{P}\right)=S(r, f)=S(r, P)
$$

by the conditions (2.4) and (4.11). Hence it follows from (4.13) and (4.14) that

$$
\bar{N}(r, P)+\bar{N}\left(r, \frac{1}{P}\right)=S(r, P) .
$$

This completes the proof of the theorem.

\section{Further remarks}

In this section, several comparisons between our main results and previous results are given as follows:

Remark 1. In our Theorem 2.1, if we take $n=1$, then we get a result which is similar to the one of L. R. Sons stated in the introduction. If $\bar{d}(P)=\underline{d}(P)$, i.e., $P[f]$ is homogeneous, then we have the Theorem A. In addition, if $\underline{d}(P)=0$, then we get the Theorem B.

Remark 2. In 1986, H. S. Gopalakrishna and S. S. Bhoosnurmath [2] obtained the results $\sigma(P)=\sigma(f)$ and $\bar{N}(r, P)+\bar{N}\left(r, \frac{1}{P}\right)=S(r, P)$ by assuming that the condition (2.4) holds and $P[f]$ is a homogeneous differential polynomial in $f$ which does not reduce to a constant. Thus it is easy to see that if the differential polynomial is homogeneous in the Theorem 2.2, then we have a result which is similar to the result obtained by them.

\section{Acknowledgement}

The first author thanks the University Grants Commission for the award of Teacher Fellowship under Faculty Improvement Programme.

\section{References}

[1] W. Doeringer, Exceptional values of differential polynomials, Pacific J. Math., 98 (1982), 55-62.

[2] H. S. Gopalakrishna and S. S. Bhoosnurmath, On the distribution of values of differential polynomials, Indian J. Pure Appl. Math. 17 (1986), 367-372.

[3] W. K. Hayman, Picard values of meromorphic functions and their derivatives, Ann. of Math. 70 (1959), 9-42.

[4] W. K. Hayman, Meromorphic functions, Clarendon Press, Oxford, 1975 (with appendix).

[5] H. Milloux, Les fonctions méromorphes et leurs dérivées, Pairs, 1940. 
[6] A. P. Singh, On order of homogeneous differential polynomials, Indian J. Pure Appl. Math. 16 (1985), 791-795.

[7] A. P. Singh and R. S. Dhar, On orders of differential polynomials, Proc. Nat. Acad. Sci. India Sect. A 64 (1994), 365-372.

[8] L. R. Sons, Deficiencies of monomials, Math. Z. 111 (1969), 53-68.

[9] L. Yang, Value Distribution Theory (Chinese), Science Press Beijing, 1993.

Department of Mathematics, Karnatak University, Dharwad, India.

E-mail: meelind2000@yahoo.co.in

Department of Mathematics, United Christian College, 7-11, Tong Yam Street, Tai Hang Tung, Kowloon, Hong Kong, China.

E-mail: kitwing@hotmail.com 